\title{
Next-to-leading tail-induced spin-orbit effects in the gravitational radiation flux of compact binaries
}

\author{
Sylvain Marsat, 1, *Alejandro Bohé, 2, †Luc Blanchet, 1, 用and Alessandra Buonanno 3, 8 \\ ${ }^{1} \mathcal{G} \mathbb{R} \varepsilon \mathbb{C O}$ Institut d'Astrophysique de Paris - UMR 7095 du CNRS, \\ Université Pierre $\&$ Marie Curie, $98^{\text {bis }}$ boulevard Arago, 75014 Paris, France \\ ${ }^{2}$ Departament de Física, Universitat de les Illes Balears, \\ Crta. Valldemossa km 7.5, E-07122 Palma, Spain \\ ${ }^{3}$ Maryland Center for Fundamental Physics 8 Joint Space-Science Center, \\ Department of Physics, University of Maryland, College Park, MD 20742, USA
}

(Dated: September 10, 2018)

\begin{abstract}
The imprint of non-linearities in the propagation of gravitational waves - the tail effect — is responsible for new spin contributions to the energy flux and orbital phasing of spinning black hole binaries. The spin-orbit (linear in spin) contribution to this effect is currently known at leading post-Newtonian order, namely 3PN for maximally spinning black holes on quasi-circular orbits. In the present work, we generalize these tail-originated spin-orbit terms to the next-to-leading $4 \mathrm{PN}$ order. This requires in particular extending previous results on the dynamical evolution of precessing compact binaries. We show that the tails represent the only spin-orbit terms at that order for quasi-circular orbits, and we find perfect agreement with the known result for a test particle around a Kerr black hole, computed by perturbation theory. The BH-horizon absorption terms have to be added to the PN result computed here. Our work completes the knowledge of the spin-orbit effects to the phasing of compact binaries up to the $4 \mathrm{PN}$ order, and will allow the building of more faithful PN templates for the inspiral phase of black hole binaries, improving the capabilities of ground-based and space-based gravitational wave detectors.

PACS numbers: $04.25 . \mathrm{Nx}, 04.25 . \mathrm{dg}, 04.30 .-\mathrm{w}$
\end{abstract}

\footnotetext{
* marsat@iap.fr

$\dagger$ alejandro.bohe@uib.es

$\ddagger$ blanchet@iap.fr

§ buonanno@umd.edu
} 


\section{INTRODUCTION}

This work is the continuation of our series of papers [1-3], where we computed the nextto-next-to-leading spin-orbit effects in the dynamics and gravitational radiation of black hole binary systems. These next-to-next-to-leading contributions are $2 \mathrm{PN} \sim 1 / c^{4}$ orders beyond the leading spin-orbit effect which arises at $1.5 \mathrm{PN} \sim 1 / c^{3}$ order - thus being of absolute 3.5PN $\sim 1 / c^{7}$ order ${ }^{1}$ More specifically, we derived in Ref. [1] the corresponding contributions to the equations of motion in harmonic coordinates, and proved the equivalence of our result with the one obtained previously within the ADM Hamiltonian formalism [4, 5]. In Ref. [2] we presented explicit results for the conserved integrals of the motion, the precession equations for the spins and the near-zone PN metric. In Ref. [3] we obtained the corresponding results for the radiative multipole moments, energy flux and orbital phasing. In the present paper, we address the computation of the tail contributions to the emitted energy flux and to the phasing of the binary to the next-to-leading order, which corresponds to $4 \mathrm{PN} \sim 1 / c^{8}$, thus extending the computation performed in Ref. [6] where these tail effects were obtained at the leading $3 \mathrm{PN} \sim 1 / c^{6}$ order. Hereafter we shall refer to the works [6] and [3] as Papers I \& II respectively.

The above PN counting for spin effects refers to maximally spinning black holes. In keeping with the conventions used in Papers I \& II, we use as a spin variable $S \equiv c S_{\text {true }}=G m^{2} \chi$, where $m$ is the compact body's mass and $S_{\text {true }}$ has the dimension of an angular momentum, with $\chi$ the dimensionless spin parameter, which is 1 for a maximally spinning Kerr black hole. With this definition, the spins of the two bodies are considered as "Newtonian" quantities, and all spin effects include (at least) an explicit 1/c factor with respect to non-spinning effects. One should keep in mind that the spin-orbit effects will be formally half a PN order smaller - and our computations will thus be half a PN order more accurate — for non-maximally spinning objects like neutron stars.

Computing high-order PN corrections to the gravitational waveform emitted by compact binaries permits a better comparison with numerical relativity results, and improves the accuracy of the templates that will be used in the data analysis of gravitational wave groundbased detectors such as LIGO, Virgo and KAGRA, and, further ahead, space-based LISA-like detectors. Including the effects of spins is essential, as recent astrophysical evidence indicates that stellar-mass black holes [7-11] and supermassive black holes [12 14] (see Ref. [15] for a review) can be generically close to maximally spinning. The presence of spins crucially affects the dynamics of the binary, in particular leading to orbital plane precession if they are not aligned with the orbital angular momentum (see for instance [16, 17]), and to strong modulations in the observed signal frequency and phase.

The spin-orbit effects have been known at the leading order $(1.5 \mathrm{PN})$ since the seminal works [18 21]. They have been extended more recently to the next-to-leading order (2.5PN) in Refs. [22 26] for the equations of motion and in Ref. [27] for the radiation field. Spin-spin interactions are also known: see Refs. [21, 28 30] for the leading (2PN) order in the equations of motion and radiation field; [31 35] for the next-to-leading (3PN) order in the equations of motion; and [36, 37] for the next-to-next-to-leading $(4 \mathrm{PN})$ order in the equations of motion for the coupling of different spins.

In line with Papers I \& II, we use the multipolar post-Newtonian approach to gravitational radiation, which combines a multipolar-post-Minkowskian expansion for the vacuum field in the exterior of the matter source [38], together with a matching to the post-Newtonian field

\footnotetext{
${ }^{1}$ As usual we refer to $n \mathrm{PN}$ as the post-Newtonian (PN) terms with formal order $\mathcal{O}\left(c^{-2 n}\right)$.
} 
inside the source [39] (see Ref. [40] for a review). In that formalism, the tails, which are physically due to the backscatter of linear waves from the curvature of space-time generated by the total mass of the source, appear as integrals over the past of the source, which enter the relationships between the radiative multipole moments which are observed at infinity from the source, and the source-rooted multipole moments.

From a data analysis point of view, such tail contributions are very important features of the waveform of inspiralling compact binaries, and will likely be decoded by the next generation of detectors, i.e. the advanced versions of LIGO and Virgo on ground, and by the future LISA-like detectors in space. More specifically, we shall show, using an estimate of the number of cycles of the waveform in the appropriate frequency bands (based on the Taylor T2 approximant), that the spin-orbit tail contribution at leading and next-to-leading orders is relevant to the future data analysis of these detectors and should be included in the gravitational wave templates.

The plan of this paper is as follows. In Sec. [I] we briefly recall the general formalism for gravitational wave generation and the various types of contributions to the waveform and flux, including the tails. We also show that, at the $4 \mathrm{PN}$ order and at the spin-orbit level for circular orbits, the only contribution to the flux originates from the tails. In Sec. [II] we describe the dynamics of the precessing binary, and we give an explicit analytical solution for the precession, formally valid up to any $\mathrm{PN}$ order but neglecting radiation reaction and limited to the spin-orbit level. In Sec. [V] we provide the necessary expressions for the source moments (taken from Paper II), explain our calculations of the tail integrals both in the Fourier and time domains, and give our final results for the emitted flux and the orbital phasing of the binary. Appendix A provides some further technical explanations.

\section{GRAVITATIONAL WAVE TAILS IN THE ENERGY FLUX}

\section{A. Radiative versus source multipole moments}

The total gravitational-wave energy flux, emitted in all directions around the source, is

$$
\mathcal{F} \equiv\left(\frac{\mathrm{d} \mathcal{E}}{\mathrm{d} t}\right)^{\mathrm{GW}} \equiv\left(\int \mathrm{d} \Omega \frac{\mathrm{d} \mathcal{E}}{\mathrm{d} t \mathrm{~d} \Omega}\right)^{\mathrm{GW}},
$$

where $\mathcal{E}$ denotes the energy carried away in the gravitational waves. In the most general case the flux is given as an infinite series of multipolar contributions (starting at the quadrupole level $\ell=2$ ), by [41]

$$
\mathcal{F}=\sum_{\ell=2}^{+\infty} \frac{G}{c^{2 \ell+1}}\left[\frac{(\ell+1)(\ell+2)}{(\ell-1) \ell \ell !(2 \ell+1) ! !} U_{L}^{(1)} U_{L}^{(1)}+\frac{4 \ell(\ell+2)}{c^{2}(\ell-1)(\ell+1) !(2 \ell+1) ! !} V_{L}^{(1)} V_{L}^{(1)}\right] .
$$

The radiative multipole moments $U_{L}$ with mass-type and $V_{L}$ with current-type parametrize (by definition) the asymptotic transverse-traceless spatial waveform at leading order in the distance to a general matter source. Consequently they also parametrize the various gravitational wave fluxes like the energy flux..$^{2}$ The radiative moments are functions of the retarded

2 The notation for multi-indices and symmetric-trace-free (STF) tensors like $U_{L}$ and $V_{L}$ is the same as in Papers I \& II. Thus we denote by $L=i_{1} \cdots i_{\ell}$ a multi-index composed of $\ell$ multipolar spatial indices $i_{1}, \cdots, i_{\ell}$ ranging from 1 to 3 . In the case of summed-up (dummy) multi-indices $L$, we do not write the $\ell$ summations from 1 to 3 over their indices. Time derivatives are indicated with a superscript $(n)$. 
time $T_{R} \equiv T-R / c$ in a radiative coordinate system which by definition is a system for which $T_{R}$ coincides with a null coordinate asymptotically in the limit $R \equiv\left|X^{i}\right| \rightarrow \infty$.

In order to define a wave generation formalism, the radiative moments $U_{L}\left(T_{R}\right)$ and $V_{L}\left(T_{R}\right)$ are to be related to the matter content of the source. This is done in two steps. First, they are expressed in terms of some "canonical" multipole moments $M_{L}$ and $S_{L}$. The relations between the radiative moments $U_{L}, V_{L}$ and the canonical ones $M_{L}, S_{L}$ encode the nonlinearities in the wave propagation between the source and the detector. Those relations are re-expanded in a $\mathrm{PN}$ approximation and are then seen to contain, at the leading 1.5PN order, the contribution of the gravitational-wave tails, which take the form of "hereditary" type integrals, formally depending on all the infinite past of the source. Explicitly we have [42, 43]

$$
\begin{aligned}
& U_{L}\left(T_{R}\right)=M_{L}^{(\ell)}\left(T_{R}\right)+\frac{2 G M}{c^{3}} \int_{0}^{+\infty} \mathrm{d} \tau M_{L}^{(\ell+2)}\left(T_{R}-\tau\right)\left[\ln \left(\frac{\tau}{2 \tau_{0}}\right)+\kappa_{\ell}\right]+\mathcal{O}\left(\frac{1}{c^{5}}\right), \\
& V_{L}\left(T_{R}\right)=S_{L}^{(\ell)}\left(T_{R}\right)+\frac{2 G M}{c^{3}} \int_{0}^{+\infty} \mathrm{d} \tau S_{L}^{(\ell+2)}\left(T_{R}-\tau\right)\left[\ln \left(\frac{\tau}{2 \tau_{0}}\right)+\pi_{\ell}\right]+\mathcal{O}\left(\frac{1}{c^{5}}\right) .
\end{aligned}
$$

The constant ADM mass $M$ of the source (or mass monopole) is responsible for the backscattering of the linear waves producing tails. The logarithmic kernels of the tail integrals involve a freely specifiable time scale $\tau_{0}$ entering the relation between the radiative time $T_{R}$ and the corresponding retarded time $t_{r} \equiv t-r / c$ in harmonic coordinates:

$$
T_{R}=t_{r}-\frac{2 G M}{c^{3}} \ln \left(\frac{r}{c \tau_{0}}\right) .
$$

The numerical constants $\kappa_{\ell}$ and $\pi_{\ell}$ appearing in Eqs. 2.3) (which depend on the choice of harmonic coordinates used to cover the source) are given by

$$
\begin{aligned}
& \kappa_{\ell}=\frac{2 \ell^{2}+5 \ell+4}{\ell(\ell+1)(\ell+2)}+\sum_{k=1}^{\ell-2} \frac{1}{k}, \\
& \pi_{\ell}=\frac{\ell-1}{\ell(\ell+1)}+\sum_{k=1}^{\ell-1} \frac{1}{k} .
\end{aligned}
$$

Since spin-orbit effects start at order $\mathcal{O}\left(c^{-3}\right)$ in the mass-type moments and at order $\mathcal{O}\left(c^{-1}\right)$ in the current-type moments [27], one can easily check that in order to obtain the spin-orbit terms at $4 \mathrm{PN}$ in the flux we need only the tails in the mass and current quadrupole moments $U_{i j}$ and $V_{i j}$ (i.e. having $\ell=2$ ), and these will have to be computed at $1 \mathrm{PN}$ relative order, and in the mass and current octupoles $U_{i j k}$ and $V_{i j k}(\ell=3)$, to be computed at Newtonian order.

As a second step, the canonical moments $M_{L}$ and $S_{L}$ are related to a particular set of six source-rooted multipole moments, that admit explicit analytic closed form expressions as integrals over the matter and gravitational fields in the source [39]. This new set of moments can be divided into two "source" multipole moments $I_{L}$ and $J_{L}$ (mass-type and current-type), and four so-called "gauge" multipole moments $W_{L}, X_{L}, Y_{L}, Z_{L}$ which play a role only at high post-Newtonian orders. For our purpose, it will be sufficient to know that $M_{L}$ and $S_{L}$ coincide with the source moments $I_{L}$ and $J_{L}$ up to small PN remainders $\mathcal{O}\left(c^{-5}\right)$ :

$$
M_{L}=I_{L}+\mathcal{O}\left(\frac{1}{c^{5}}\right)
$$




$$
S_{L}=J_{L}+\mathcal{O}\left(\frac{1}{c^{5}}\right) .
$$

The PN remainders $\mathcal{O}\left(c^{-5}\right)$ in both Eqs. 2.3) and (2.6) contain different sorts of nonlinear interactions between (time derivatives of the) multipole moments. These can be divided into hereditary terms [42], which involve various integrals over the whole past of the multipole moments like in the tails (2.3), and instantaneous terms which depend only on the current values of the multipole moments at instant $T_{R}$. Here our nomenclature refers to terms which are hereditary or instantaneous functionals of the source and gauge moments $I_{L}, J_{L}, W_{L}, \cdots, Z_{L}$ (i.e. after due replacement of the canonical moments $M_{L}, S_{L}$ in terms of $\left.I_{L}, J_{L}, \cdots, Z_{L}\right)$. For instance the hereditary terms in Eqs. (2.3) comprise at order $\mathcal{O}\left(c^{-5}\right)$ the so-called non-linear memory effect which is a quadratic interaction between multipole moments, ${ }^{3}$ and, at order $\mathcal{O}\left(c^{-6}\right)$, the so-called tail-of-tail term which is cubic. The nonlinear memory integral is simply given by an anti-derivative of an instantaneous term, while the tail-of-tail involves a logarithmic kernel similar to the one in Eqs. (2.3) — although more complicated. In addition there are many couplings between moments which are just instantaneous; see the explicit formulas given in Refs. [44, 45]. Recalling that spin-orbit contributions bring at least an additional factor $1 / c$, we see that we should in principle take into account all these instantaneous corrections up to the order $\mathcal{O}\left(c^{-7}\right)$ in the mass quadrupole moment $U_{i j}$ and $\mathcal{O}\left(c^{-5}\right)$ in the current quadrupole moment $V_{i j}$ (as given in Refs. [44, 45]).

\section{B. Contributions to the flux for circular orbits}

We now restrict ourselves to compact binaries whose orbit has been circularized by the emission of gravitational radiation, so that it can be considered as quasi-circular. That is to say, the orbital elements (except for precession effects due to the presence of spins) are assumed to vary only on long timescales, because of radiation reaction. This restriction to quasi-circular orbits will also allow us to model simply the dynamics of the binary in the past and therefore to compute the hereditary tail integrals (2.3). Anticipating on the notation used for compact binaries in the following section, the orbital separation $r$ and orbital frequency $\omega$ will thus be assumed to vary according to ${ }^{4}$

$$
\dot{r}=\mathcal{O}\left(\frac{1}{c^{5}}\right), \quad \dot{\omega}=\mathcal{O}\left(\frac{1}{c^{5}}\right) .
$$

An important point is that, when restricting the calculation to quasi-circular orbits, purely instantaneous terms cannot give any spin-orbit contribution at 4PN order in the energy flux (2.2). We show this fact by a simple dimensional analysis. Indeed, we can write the general structure of such instantaneous terms in the flux as

$$
(\mathcal{F})_{\text {inst }} \sim \sum \frac{(G m)^{n}}{c^{a} r^{k}}(n, v, S)\left(\boldsymbol{v}^{2}\right)^{p}(\boldsymbol{n} \cdot \boldsymbol{v})^{q},
$$

where $m$ is any of the two masses in the binary system, $\boldsymbol{v}^{2} \equiv \dot{r}^{2}+r^{2} \omega^{2}$ is the squared Euclidean norm of the relative velocity between the two bodies, and $\boldsymbol{n} \cdot \boldsymbol{v} \equiv \dot{r}$ is the Euclidean

\footnotetext{
${ }^{3}$ Actually this effect appears only in the $\mathcal{O}\left(c^{-5}\right)$ correction of the mass-type radiative multipole moment $U_{L}$, but not in the current-type radiative moment $V_{L}$.

${ }^{4}$ As we shall check later the orbital frequency for circular orbits is constant at linear order in the spins.
} 
scalar product between the unit separation vector between the two particles and their relative velocity. We are assuming that the expression of the flux is given in the frame of the center of mass. There is no dependence on the relative acceleration since it is supposed to have been consistently replaced by the equations of motion - the normal practice in PN approximations. Note that since we are dealing with instantaneous (non-hereditary) terms, the velocity $\boldsymbol{v}$ and unit direction $\boldsymbol{n}$ are taken at the same time, which is the current instant $T_{R}$; there is no integration over some intermediate time in between which would couple together some of these vectors at different instants.

The dependence on the two spin vectors can only arise through the mixed product $(n, v, S) \equiv \varepsilon_{i j k} n^{i} v^{j} S^{k}$, where $S^{i}$ denotes any of the two spin vectors, with any of the usual conventions adopted for the spin vectors. This is easily proven if one remembers that the spin vectors are actually pseudo-vectors with respect to parity transformations, while the flux must be a scalar, i.e. not a pseudo-scalar. In Eq. (2.8) we are considering only terms linear in the spins, neglecting quadratic spin-spin coupling terms.

As recalled in the Introduction, with our convention used in this series of papers [1] the dimension of the spin tensor and of all spin variables are that of an angular momentum times the speed of light $c$. With that convention it is easy to check that in order for the flux to have the correct dimension of a power (energy per unit time), we need $k=n+2$ and $2 p+q+2 n=a$. For a $4 \mathrm{PN}$ term, we should have $a=13$ in Eq. (2.8) because this corresponds to $4 \mathrm{PN} \sim 1 / c^{8}$ beyond the leading radiation reaction at $2.5 \mathrm{PN} \sim 1 / c^{5}$ order, hence $6.5 \mathrm{PN} \sim 1 / c^{13}$ absolute order. Hence we deduce that $q=13-2 p-2 n$. The point is that $q$ should be an odd integer for a $4 \mathrm{PN}$ term, and thus that this term contains at least one factor $\boldsymbol{n} \cdot \boldsymbol{v}$. Since for quasi-circular orbits we have $\boldsymbol{n} \cdot \boldsymbol{v}=\dot{r}=\mathcal{O}\left(c^{-5}\right)$, the real order of magnitude of this term is very small, being at least $6.5 \mathrm{PN}$ (or 9PN absolute).

Thus, we have proved that instantaneous terms (i.e. which do not involve any hereditary integral) will be negligible for our purposes. Now, let us show that the only truly hereditary integrals which can contribute spin-orbit terms at 4PN order in the flux are the tails given in (2.3). The tail-of-tail term which appears at order $\mathcal{O}\left(c^{-6}\right)$ in $U_{i j}$ involves the mass quadrupole moment, and therefore the spin-orbit contributions therein, which are $\mathcal{O}\left(c^{-3}\right)$ for mass moments, will appear only at higher order. On the other hand, we have already remarked that the non-linear memory integrals at orders $\mathcal{O}\left(c^{-5}\right)$ and $\mathcal{O}\left(c^{-7}\right)$ are given by some simple anti-derivatives. They become therefore instantaneous in the energy flux (2.2) in which all the radiative moments are differentiated with time; so the previous argument applies to such terms as well.

Our conclusion is that the only contributions coming from the spin-orbit effect at the $4 \mathrm{PN}$ order in the case of quasi-circular orbits are due to the hereditary tail integrals given in Eqs. (2.3). There are no contributions from other hereditary terms nor instantaneous ones, either coming from non-linear interactions between canonical moments in the remainders of (2.3), or from the correspondance between canonical and source and gauge moments (2.6). In particular, we can ignore the $4 \mathrm{PN}$ spin-orbit terms in the relative acceleration which is used in this calculation to order reduce the time derivatives of the moments 5 Notice that this argument about instantaneous terms shows that the arbitrary scale $\tau_{0}$ used to adimensionize the logarithmic kernel of the tail integrals $(2.3)$ will disappear from the final result as it is in factor of an instantaneous term. The same is true for the numerical constants $\kappa_{\ell}$ and $\pi_{\ell}$ which are irrelevant for this calculation. We emphasize that all these statements are limited

\footnotetext{
${ }^{5}$ Such $4 \mathrm{PN}$ spin-orbit terms in the equations of motion are instantaneous, and correspond to a $1.5 \mathrm{PN}$ spin-orbit modification of the standard 2.5PN radiation reaction force [46].
} 
to quasi-circular orbits, neglecting their possible eccentricity, and to the computation of the energy flux. They do not apply to the computation of the full waveform with its two polarizations. The two polarizations $h_{+}$and $h_{\times}$, although being scalars, depend on the direction of the source and on the polarization vectors, so the structure analogous to (2.8) is more complicated.

The calculation of hereditary integrals like the tail integrals in Eqs. (2.3) in principle requires knowing explicitly the dynamics of the binary system in the past. One must first supplement the computation with some physical assumption regarding the behaviour of the source in the infinite past. Following Refs. [42, 47] and Paper I we can assume that at very early times the binary system was formed from freely falling black holes moving initially on some hyperbolic-like orbits. This ensures that the integrals in (2.3) are convergent (see e.g. the discussion in Sec. II B of Paper I). It was then shown [42, 47] that under such an assumption the tail integrals are very weakly sensitive over the past history of the source, and can essentially be computed by inserting the current dynamics (at current time $T_{R}$ ) of the binary into the integrals - i.e. neglecting the secular changes of the orbit by radiation reaction over the past. Quite naturally, as proved in the Appendix of Ref. [47], one can proceed in that way modulo some $\mathrm{PN}$ remainder terms of the order of the radiation reaction scale, i.e. $\mathcal{O}\left(c^{-5}\right)$ and more precisely $\mathcal{O}\left(\ln c / c^{5}\right)$. Nevertheless, even if we can always neglect the evolution of the orbit by gravitational radiation in the past, one has still to worry about the details of the current dynamics which has to be plugged into the tail integrals and consistently integrated. This is dealt with in the next section.

\section{ANALYTICAL SOLUTION FOR THE SPIN-ORBIT DYNAMICS}

In this section, we present an analytical solution for the dynamics of the binary of compact spinning objects on quasi-circular orbits, including the precession effects due to the presence of the spins. This solution will be valid formally at any post-Newtonian order, if radiation reaction effects are neglected, but will be restricted to the linear order in spins. The leading order solution was already obtained in Paper I, but we shall show that the solution found there turns out to be in fact nicely valid to higher PN orders, provided that we restrict to spin-orbit contributions. To show this, we parallel the presentation given in Paper I, repeating all the necessary definitions for completeness, and pointing out where the validity of the solution can in fact be extended to higher order.

\section{A. Equations of motion and spin precession for quasi-circular orbits}

Throughout this paper, we will work in the center-of-mass frame, defined by the cancellation of the center-of-mass integral of motion $\boldsymbol{G}=0$, and we will use conserved-norm spin variables as they are defined in Ref. [2], where a systematic construction, fixing the convention, is proposed $!^{6}$ This choice allows one to write the evolution equations of the spin vectors as simple precession equations, see Eq. 3.13 below, and, as discussed in Papers

${ }^{6}$ Notice that the definition used here for the conserved-norm spin vectors is distinct from the one used in Ref. 27. However, the difference between the two variables is of order $2 \mathrm{PN}$ and vanishes in the center-of-mass frame. For reference we give here the relation between these two conserved-norm variables:

$$
\mathbf{S}_{1}=\mathbf{S}_{1}^{\mathrm{BBF}}+\frac{2 G m^{2}}{c^{4} r_{12}}\left[\left(S_{1} v_{1}\right) \boldsymbol{v}_{2}-\left(S_{1} v_{2}\right) \boldsymbol{v}_{1}\right]+\mathcal{O}\left(\frac{1}{c^{6}}\right)
$$

where $r_{12}$ is the orbital separation and $\boldsymbol{v}_{1,2}$ are the two velocities. In Paper I we worked at leading-order where all spin variables are equivalent. 
I \& II, it is crucial when applying the energy balance condition relating the emitted flux and the decrease of the orbital energy, since these variables will be secularly constant. It is convenient to introduce two combinations of the individuals spins defined by

$$
\boldsymbol{S} \equiv \boldsymbol{S}_{1}+\boldsymbol{S}_{2}, \quad \boldsymbol{\Sigma} \equiv \frac{m}{m_{2}} \boldsymbol{S}_{2}-\frac{m}{m_{1}} \boldsymbol{S}_{1},
$$

with $m \equiv m_{1}+m_{2}$ the total mass. Later we will also use the symmetric mass ratio $\nu \equiv$ $m_{1} m_{2} / m^{2}$ and the mass difference $\delta m \equiv m_{1}-m_{2}$.

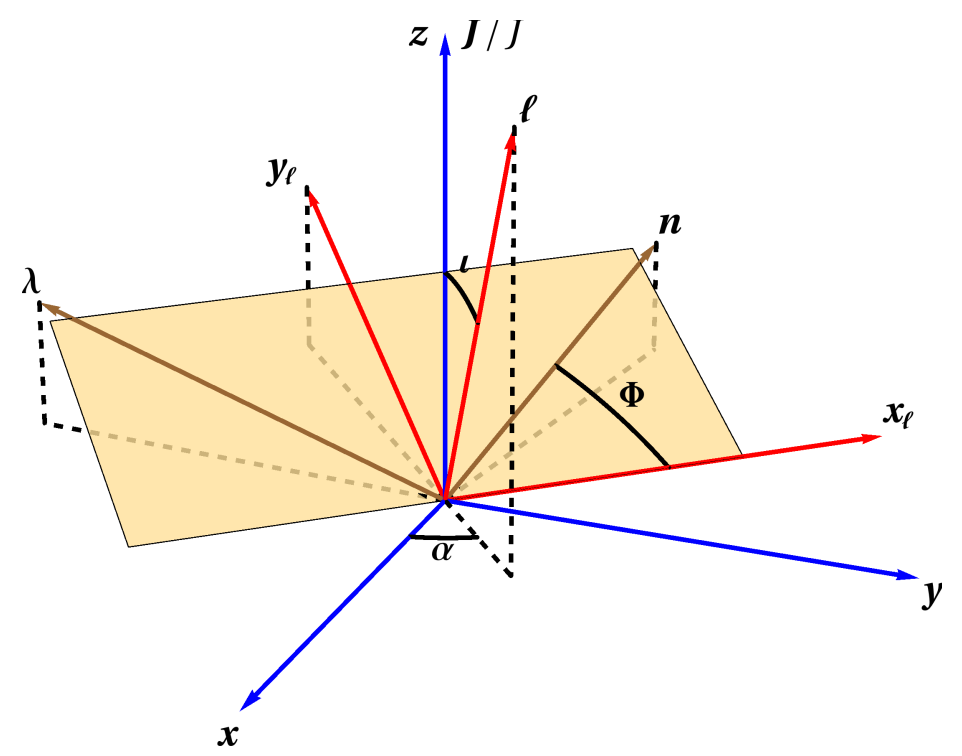

FIG. 1. Geometric definitions to describe the precessional motion of the binary, identical to the ones used in Paper I. The conserved angular momentum $\boldsymbol{J}$ gives a fixed direction $\boldsymbol{z}$, completed with two constant unit vectors $\boldsymbol{x}$ and $\boldsymbol{y}$ forming with $\boldsymbol{z}$ an orthonormal triad; $\boldsymbol{\ell}$ is the normal to the instantaneous orbital plane (shown in yellow), described by the Euler angles $\alpha, \iota$, and defines the auxiliary vectors $\boldsymbol{x}_{\ell}, \boldsymbol{y}_{\ell}$, see Eqs. (3.4). The position of the unit separation vector $\boldsymbol{n}$ defines the third Euler angle $\Phi$, and the moving triad is completed by $\boldsymbol{\lambda}=\boldsymbol{\ell} \times \boldsymbol{n}$.

In the following, we will extensively employ the total angular momentum of the system, that we denote by $\boldsymbol{J}$, and which is conserved,

$$
\frac{\mathrm{d} \boldsymbol{J}}{\mathrm{d} t}=0,
$$

neglecting radiation-reaction effects. It is customary to decompose the conserved angular momentum as $\boldsymbol{J}=\boldsymbol{L}+\boldsymbol{S} / c$, with $\boldsymbol{S}$ being specified by our choice of conserved-norm spin variables, and with $\boldsymbol{L}$ including both spin and non-spin PN contributions. We shall give $\boldsymbol{L}$ explicitly in Eq. (3.12) below for the case of circular orbits.

To describe the relative motion of the binary in the center-of-mass frame, we keep the same geometric definitions as in Paper I, which are recalled in Fig. 1. We introduce an orthonormal triad $(\boldsymbol{n}, \boldsymbol{\lambda}, \boldsymbol{\ell})$ defined as follows: $\boldsymbol{n}$ is the unit-norm separation vector, such that $\boldsymbol{x}=r \boldsymbol{n}$ with $\boldsymbol{x} \equiv \boldsymbol{y}_{1}-\boldsymbol{y}_{2}$. From the relative velocity $\boldsymbol{v} \equiv \boldsymbol{v}_{1}-\boldsymbol{v}_{2}$, we define the unit normal $\boldsymbol{\ell}$ to the instantaneous orbital plane, as $\boldsymbol{\ell}=\boldsymbol{n} \times \boldsymbol{v} /|\boldsymbol{n} \times \boldsymbol{v}|$ (excluding the head-on 
collision case). The orthonormal triad is then completed by $\boldsymbol{\lambda}=\boldsymbol{\ell} \times \boldsymbol{n}$. In the following, the components of a vector on this basis will be denoted by a subscript, for instance $A_{n} \equiv \boldsymbol{A} \cdot \boldsymbol{n}$.

Next, denoting the time derivative by a dot, the orbital angular frequency $\omega$ and precession angular frequency $\varpi$ are defined by $\dot{\boldsymbol{n}}=\omega \boldsymbol{\lambda}$ and $\dot{\boldsymbol{\ell}}=-\varpi \boldsymbol{\lambda}$ respectively. This leads to the following system of equations for the time evolution of the triad vectors, 7

$$
\begin{aligned}
\dot{\boldsymbol{n}} & =\omega \boldsymbol{\lambda}, \\
\dot{\boldsymbol{\lambda}} & =-\omega \boldsymbol{n}+\varpi \boldsymbol{\ell}, \\
\dot{\boldsymbol{\ell}} & =-\varpi \boldsymbol{\lambda} .
\end{aligned}
$$

We also introduce a fixed orthonormal basis $(\boldsymbol{x}, \boldsymbol{y}, \boldsymbol{z})$, with the $\boldsymbol{z}$ direction along the total angular momentum $\boldsymbol{J}$ (which is conserved, as we said, if we neglect radiation reaction effects). It is convenient to introduce Euler angles to mark the position of the binary with respect to this fixed basis. Two additional vectors lying in the orbital plane are defined according to

$$
\boldsymbol{x}_{\ell}=\frac{\boldsymbol{J} \times \boldsymbol{\ell}}{|\boldsymbol{J} \times \boldsymbol{\ell}|}, \quad \boldsymbol{y}_{\ell}=\boldsymbol{\ell} \times \boldsymbol{x}_{\ell},
$$

and the Euler angles $\alpha, \iota$, and $\Phi$ are defined as indicated in Fig. 1. The relation between $(\boldsymbol{n}, \boldsymbol{\lambda})$ and $\left(\boldsymbol{x}_{\ell}, \boldsymbol{y}_{\ell}\right)$ is then

$$
\begin{aligned}
& \boldsymbol{n}=\cos \Phi \boldsymbol{x}_{\ell}+\sin \Phi \boldsymbol{y}_{\ell}, \\
& \boldsymbol{\lambda}=-\sin \Phi \boldsymbol{x}_{\ell}+\cos \Phi \boldsymbol{y}_{\ell} .
\end{aligned}
$$

We also have for the inclination angle $\iota$ :

$$
\sin \iota=\frac{|\boldsymbol{J} \times \ell|}{|\boldsymbol{J}|} .
$$

Computing the product $\sin \iota \boldsymbol{x}_{\ell} \cdot(\boldsymbol{n}+\mathrm{i} \boldsymbol{\lambda})$ in two different ways, using (3.4) and (3.5), yields a relation which will be important in the following:

$$
\sin \iota e^{-\mathrm{i} \Phi}=-\mathrm{i} \frac{J_{+}}{|\boldsymbol{J}|}
$$

where we defined $J_{+} \equiv J_{n}+\mathrm{i} J_{\lambda}$. Using the derivatives of the basis vectors as given by (3.3), we arrive at the following system of equations for the time derivatives of the Euler angles:

$$
\begin{aligned}
\frac{\mathrm{d} \alpha}{\mathrm{d} t} & =\varpi \frac{\sin \Phi}{\sin \iota}, \\
\frac{\mathrm{d} \iota}{\mathrm{d} t} & =\varpi \cos \Phi, \\
\frac{\mathrm{d} \Phi}{\mathrm{d} t} & =\omega-\varpi \frac{\sin \Phi}{\tan \iota} .
\end{aligned}
$$

Notice that the only assumption we made in deriving Eqs. (3.8) was to treat the total angular momentum as a constant, that is to say neglecting the radiation reaction effects. The above

\footnotetext{
${ }^{7}$ Notice that we changed our notations with respect to Paper I; our $\varpi$ corresponding to $-\omega_{\text {prec }}$ there.
} 
relations are valid, in particular, for general orbits and not only for quasi-circular ones. They are suitable for insertion into the tail integrals modulo negligible radiation reaction corrections $\mathcal{O}\left(\ln c / c^{5}\right)$.

The general expression for the relative acceleration $\boldsymbol{a} \equiv \mathrm{d} \boldsymbol{v} / \mathrm{d} t$ decomposed in the moving frame is given by $\boldsymbol{a}=\left(\ddot{r}-r \omega^{2}\right) \boldsymbol{n}+(r \dot{\boldsymbol{\omega}}+2 \dot{\boldsymbol{r}} \omega) \boldsymbol{\lambda}+r \omega \varpi \boldsymbol{\ell}$. In the following, we will restrict ourselves to quasi-circular orbits, where we can set Eqs. 2.7) namely $\dot{r}, \dot{\omega}=\mathcal{O}\left(c^{-5}\right)$. Thus, the moving point will stay on a sphere of constant radius, and we have

$$
\boldsymbol{a}=-r \omega^{2} \boldsymbol{n}+r \omega \varpi \boldsymbol{\ell}+\mathcal{O}\left(\frac{1}{c^{5}}\right) .
$$

The component of the acceleration along $\boldsymbol{\ell}$, proportional to $\varpi=\mathcal{O}(S)$, is responsible for the slow precession of the orbital plane. All the information about the orbital dynamics of quasi-circular orbits is encoded in two equations: one relating the orbital frequency $\omega$ to the orbital separation $r$, and one relating $\varpi$ to $\omega$. As usual we introduce two dimensionless PN parameters $\gamma$ and $x$, both being of order $\mathcal{O}\left(c^{-2}\right)$ and respectively linked to $r$ and to $\omega$ by

$$
\gamma \equiv \frac{G m}{r c^{2}}, \quad x \equiv\left(\frac{G m \omega}{c^{3}}\right)^{2 / 3} .
$$

We give here $\omega$ and $\varpi$ including the spin-orbit contribution to next-to-leading order, i.e. at 2.5PN order; we include all non-spin contributions up to this order, but notice that in fact we shall only need the next-to-leading order for the non-spin terms, i.e. 1PN. We have (see e.g. Ref. [2])

$$
\begin{aligned}
\omega^{2}=\frac{G m}{r^{3}}\{1 & +\gamma(-3+\nu)+\gamma^{2}\left(6+\frac{41}{4} \nu+\nu^{2}\right)+\frac{\gamma^{3 / 2}}{G m^{2}}\left[-5 S_{\ell}-3 \frac{\delta m}{m} \Sigma_{\ell}\right] \\
& \left.+\frac{\gamma^{5 / 2}}{G m^{2}}\left[\left(\frac{45}{2}-\frac{27}{2} \nu\right) S_{\ell}+\frac{\delta m}{m}\left(\frac{27}{2}-\frac{13}{2} \nu\right) \Sigma_{\ell}\right]\right\}+\mathcal{O}\left(\frac{1}{c^{6}}\right), \quad(3.11 \mathrm{a}) \\
\varpi= & \frac{c^{3} x^{3}}{G^{2} m^{3}}\left\{\left[7 S_{n}+3 \frac{\delta m}{m} \Sigma_{n}\right]+x\left[(-3-12 \nu) S_{n}+\frac{\delta m}{m}\left(-3-\frac{11}{2} \nu\right) \Sigma_{n}\right]\right\}+\mathcal{O}\left(\frac{1}{c^{7}}\right) .
\end{aligned}
$$

In the following, we will mostly use the PN parameter $x$ instead of $\gamma$. In fact, we will write down a solution for the dynamics directly from the conserved angular momentum $\boldsymbol{J}$ without resorting to the acceleration, so that we will not use the expression of $\varpi$ as such. An important point is that, as shown in Eq. (3.11a), at linear order in the spins only the components of the conserved-norm spin vectors along $\ell$ can contribute to $\omega$. As we shall show in Eq. (3.16c) below, these components are in fact constant at linear order in spin, when neglecting radiation reaction effects. Thus we can treat the orbital frequency $\omega$ as a constant for our purposes.

The central result that encompasses the information we need for our solution of the spinorbit dynamics is the expression of the conserved angular momentum $\boldsymbol{J}$. Again, we give here its expression at 2.5PN order but the non-spin part could be truncated at $1 \mathrm{PN}$ order for our purposes. The leading-order spin contribution is just $\boldsymbol{S} / c$. Having defined $\boldsymbol{J}=\boldsymbol{L}+\boldsymbol{S} / c$, we have then (see e.g. [2])

$$
\boldsymbol{L}=\frac{G m^{2} \nu}{c x^{1 / 2}}\left\{\boldsymbol{\ell}\left[1+x\left(\frac{3}{2}+\frac{1}{6} \nu\right)+x^{2}\left(\frac{27}{8}-\frac{19}{8} \nu+\frac{1}{24} \nu^{2}\right)\right]\right.
$$




$$
\begin{gathered}
+\frac{x^{3 / 2}}{G m^{2}}\left(\boldsymbol{\ell}\left[-\frac{35}{6} S_{\ell}-\frac{5}{2} \frac{\delta m}{m} \Sigma_{\ell}\right]+\boldsymbol{\lambda}\left[-3 S_{\lambda}-\frac{\delta m}{m} \Sigma_{\lambda}\right]+\boldsymbol{n}\left[\frac{1}{2} S_{n}+\frac{1}{2} \frac{\delta m}{m} \Sigma_{n}\right]\right) \\
+\frac{x^{5 / 2}}{G m^{2}}\left(\boldsymbol{\ell}\left[\left(-\frac{77}{8}+\frac{427}{72} \nu\right) S_{\ell}+\frac{\delta m}{m}\left(-\frac{21}{8}+\frac{35}{12} \nu\right) \Sigma_{\ell}\right]\right. \\
+\boldsymbol{\lambda}\left[\left(-\frac{7}{2}+3 \nu\right) S_{\lambda}+\frac{\delta m}{m}\left(-\frac{1}{2}+\frac{4}{3} \nu\right) \Sigma_{\lambda}\right] \\
\left.\left.+\boldsymbol{n}\left[\left(\frac{11}{8}-\frac{19}{24} \nu\right) S_{n}+\frac{\delta m}{m}\left(\frac{11}{8}-\frac{5}{12} \nu\right) \Sigma_{n}\right]\right)\right\}+\mathcal{O}\left(\frac{1}{c^{6}}\right) .
\end{gathered}
$$

The use of Euclidean conserved-norm spin vectors allows us to write their evolution equations as ordinary precession equations (with $A=1,2$ )

$$
\frac{\mathrm{d} \boldsymbol{S}_{A}}{\mathrm{~d} t}=\boldsymbol{\Omega}_{A} \times \boldsymbol{S}_{A}
$$

As already argued in Paper II, the precession vectors $\Omega_{A}$ are necessarily directed along $\ell$ at linear order in spin, so we write $\Omega_{A} \equiv \Omega_{A} \ell$. We have $\Omega_{A}=\mathcal{O}\left(c^{-2}\right)$, and the expression for $\Omega_{1}$ reads

$$
\Omega_{1}=\omega x\left\{\left(\frac{3}{4}+\frac{1}{2} \nu-\frac{3}{4} \frac{\delta m}{m}\right)+x\left[\frac{9}{16}+\frac{5}{4} \nu-\frac{1}{24} \nu^{2}+\frac{\delta m}{m}\left(-\frac{9}{16}+\frac{5}{8} \nu\right)\right]\right\}+\mathcal{O}\left(\frac{1}{c^{6}}\right)
$$

with $\Omega_{2}$ being obtained by replacing $\delta m \rightarrow-\delta m$. Using the time derivatives of the basis vectors 3.3 and the fact that $\Omega_{A} \propto \boldsymbol{\ell}$, the exact evolution equations of the components of the spins are obtained as

$$
\begin{aligned}
\frac{\mathrm{d} S_{n}^{A}}{\mathrm{~d} t} & =\left(\omega-\Omega_{A}\right) S_{\lambda}^{A}, \\
\frac{\mathrm{d} S_{\lambda}^{A}}{\mathrm{~d} t} & =-\left(\omega-\Omega_{A}\right) S_{n}^{A}+\varpi S_{\ell}^{A}, \\
\frac{\mathrm{d} S_{\ell}^{A}}{\mathrm{~d} t} & =-\varpi S_{\lambda}^{A},
\end{aligned}
$$

which readily translate, at linear order in spin, into

$$
\begin{aligned}
\frac{\mathrm{d} S_{n}^{A}}{\mathrm{~d} t} & =\left(\omega-\Omega_{A}\right) S_{\lambda}^{A}, \\
\frac{\mathrm{d} S_{\lambda}^{A}}{\mathrm{~d} t} & =-\left(\omega-\Omega_{A}\right) S_{n}^{A}+\mathcal{O}\left(S^{2}\right), \\
\frac{\mathrm{d} S_{\ell}^{A}}{\mathrm{~d} t} & =\mathcal{O}\left(S^{2}\right) .
\end{aligned}
$$

We see, as stated before, that the spin components along $\ell$ are constant, and so is the orbital frequency $\omega$ given by $3.11 \mathrm{a}$. 


\section{B. Analytical solution for the spin-orbit dynamics}

We now turn to the derivation of the explicit solution for the dynamics of the binary. We show that two relations from Paper I which were indicated to be valid neglecting higher $\mathrm{PN}$ terms of order $\mathcal{O}\left(c^{-4}\right)$ are in fact valid formally to any $\mathrm{PN}$ order, neglecting radiation reaction and working at linear order in spin. First, considering Eqs. (3.8), we see that

$$
\frac{\mathrm{d}(\Phi+\alpha)}{\mathrm{d} t}=\omega+\varpi \sin \Phi \frac{1-\cos \iota}{\sin \iota}=\omega+\mathcal{O}\left(S^{2}\right)
$$

since both the inclination angle $\iota$ and the precession frequency $\varpi$ are of order $\mathcal{O}(S)$. Thus we arrive at

$$
\Phi+\alpha=\phi+\mathcal{O}\left(S^{2}\right)
$$

introducing the "carrier" phase $\phi$ as

$$
\phi \equiv \int \mathrm{d} t \omega=\omega\left(t-t_{0}\right)+\phi_{0},
$$

with $\phi_{0}$ the reference phase at some time $t_{0}$. Secondly, we turn to Eq. (3.6). From a structural argument already presented in Paper II, the non-spin part of the angular momentum must be directed along $\boldsymbol{\ell}$, since it is a pseudo-vector built only from the vectors $\boldsymbol{n}$ and $\boldsymbol{\lambda}$. Note that this is valid in fact for general orbits and not only for circular ones. This means that the components of the angular momentum along $\boldsymbol{n}$ and $\boldsymbol{\lambda}$ come only from the presence of spins, i.e. $J_{+}=\mathcal{O}(S)$, as can be seen explicitly on (3.12). Thus, using also (3.18), we have

$$
\begin{aligned}
\sin \iota e^{-\mathrm{i} \Phi} & =-\mathrm{i} \frac{J_{+}}{\left|\boldsymbol{L}_{\mathrm{NS}}\right|}+\mathcal{O}\left(S^{2}\right), \\
\sin \iota e^{\mathrm{i} \alpha} & =-\mathrm{i} \frac{J_{+}}{\left|\boldsymbol{L}_{\mathrm{NS}}\right|} e^{\mathrm{i} \phi}+\mathcal{O}\left(S^{2}\right),
\end{aligned}
$$

with $\boldsymbol{L}_{\mathrm{NS}}$ denoting the non-spin part of $\boldsymbol{L}$ ( or $\left.\boldsymbol{J}\right)$. We will see later that these relations, together with the post-Newtonian expansion of the angular momentum which is given by (3.12) and of the spin precession frequencies (3.14), are the only ones we will need to write down our dynamical solution.

If we introduce an arbitrary reference time $t_{0}$, say the same as in Eq. (3.19), and relate each of the triads $(\boldsymbol{n}, \boldsymbol{\lambda}, \boldsymbol{\ell})$ at time $t$ and $\left(\boldsymbol{n}_{0}, \boldsymbol{\lambda}_{0}, \boldsymbol{\ell}_{0}\right)$ at time $t_{0}$ to the fixed triad $(\boldsymbol{x}, \boldsymbol{y}, \boldsymbol{z})$, and then eliminate the triad $(\boldsymbol{x}, \boldsymbol{y}, \boldsymbol{z})$, one obtains

$$
\begin{aligned}
\boldsymbol{n}=\cos & \left(\phi-\phi_{0}\right) \boldsymbol{n}_{0}+\sin \left(\phi-\phi_{0}\right) \boldsymbol{\lambda}_{0} \\
& +\left(\sin \iota \sin (\phi-\alpha)-\sin \iota_{0} \sin \left(\phi-\alpha_{0}\right)\right) \boldsymbol{\ell}_{0}+\mathcal{O}\left(S^{2}\right), \\
\boldsymbol{\lambda}=- & \sin \left(\phi-\phi_{0}\right) \boldsymbol{n}_{0}+\cos \left(\phi-\phi_{0}\right) \boldsymbol{\lambda}_{0} \\
& +\left(\sin \iota \cos (\phi-\alpha)-\sin \iota_{0} \cos \left(\phi-\alpha_{0}\right)\right) \boldsymbol{\ell}_{0}+\mathcal{O}\left(S^{2}\right), \\
\boldsymbol{\ell}=\boldsymbol{\ell}_{0} & +\left(\sin \iota \sin \left(\alpha-\phi_{0}\right)-\sin \iota_{0} \sin \left(\alpha_{0}-\phi_{0}\right)\right) \boldsymbol{n}_{0} \\
& +\left(-\sin \iota \cos \left(\alpha-\phi_{0}\right)+\sin \iota_{0} \cos \left(\alpha_{0}-\phi_{0}\right)\right) \boldsymbol{\lambda}_{0}+\mathcal{O}\left(S^{2}\right),
\end{aligned}
$$

where we used (3.18) again together with $\cos \iota=1+\mathcal{O}\left(S^{2}\right)$. The previous result can be reformulated in a more compact form if we introduce the complex null vector $\boldsymbol{m} \equiv \frac{1}{\sqrt{2}}(\boldsymbol{n}+\mathrm{i} \boldsymbol{\lambda})$ 
and its complex conjuguate $\overline{\boldsymbol{m}}$. The normalization is chosen so that $\boldsymbol{m} \cdot \overline{\boldsymbol{m}}=1$. In terms of these vectors, the result (3.21) now becomes:

$$
\begin{gathered}
\boldsymbol{m}=e^{-\mathrm{i}\left(\phi-\phi_{0}\right)} \boldsymbol{m}_{0}+\frac{\mathrm{i}}{\sqrt{2}}\left(\sin \iota e^{\mathrm{i} \alpha}-\sin \iota_{0} e^{\mathrm{i} \alpha_{0}}\right) e^{-\mathrm{i} \phi} \boldsymbol{\ell}_{0}+\mathcal{O}\left(S^{2}\right), \\
\boldsymbol{\ell}=\boldsymbol{\ell}_{0}+\left[\frac{\mathrm{i}}{\sqrt{2}}\left(\sin \iota e^{-\mathrm{i} \alpha}-\sin \iota_{0} e^{-\mathrm{i} \alpha_{0}}\right) e^{\mathrm{i} \phi_{0}} \boldsymbol{m}_{0}+\text { c.c. }\right]+\mathcal{O}\left(S^{2}\right),
\end{gathered}
$$

and we see that the precession effects in the dynamical solution for the evolution of the basis vectors $(\boldsymbol{n}, \boldsymbol{\lambda}, \boldsymbol{\ell})$, which are represented by the second term in the above equations, are all encompassed in the combination $\sin \iota e^{\mathrm{i} \alpha}$ and its complex conjugate $\sin \iota e^{-\mathrm{i} \alpha}$, which is given in terms of the spin and non-spin contributions to the angular momentum by Eq. (3.20b). Now our program is to insert the latter solution for the dynamics, Eqs. (3.21) or (3.22), into the tail integrals $(2.3)$. For that purpose it is convenient to think of $t_{0}$ as being the current retarded time $T_{R}$ and to look at the orbital evolution backwards in time.

On the other hand, the solution of the evolution equations (3.16) for the components of the spins is readily obtained as

$$
\begin{aligned}
S_{n}^{A}+\mathrm{i} S_{\lambda}^{A} & =S_{\perp}^{A} e^{-\mathrm{i} \psi_{A}}+\mathcal{O}\left(S^{2}\right), \\
S_{\ell}^{A} & =S_{\|}^{A}+\mathcal{O}\left(S^{2}\right),
\end{aligned}
$$

in which we have introduced the two integration constants $S_{\perp}^{A}$ and $S_{\|}^{A}$, and where the two spin phases are defined by

$$
\psi_{A}=\left(\omega-\Omega_{A}\right)\left(t-t_{0}\right)+\psi_{0}^{A},
$$

with $\psi_{0}^{A}$ the phases at the reference time $t_{0}$.

We are now able to analyze in more detail the dependence on time of the solution for the basis vectors and for the spins. In Eq. (3.20), $\left|\boldsymbol{L}_{\mathrm{NS}}\right|$ is simply a constant, and $J_{+}$depends on the spin components $S_{n}^{A}, S_{\lambda}^{A}$ which are given by Eqs. (3.23) and (3.24). Thus, we see that the complete dependence in time in the triad $(\boldsymbol{n}, \boldsymbol{\lambda}, \boldsymbol{\ell})$, at linear order in spin, takes the simple form of complex exponentials $e^{ \pm \mathrm{i} \omega}$ and $e^{ \pm \mathrm{i} \psi_{A}}$, so that the general structure of the time-dependent part of any product or combination of the latter basis vectors and of spin vectors is of the type (see also Paper I):

$$
e^{\mathrm{i}\left(m \omega+p \Omega_{1}+q \Omega_{2}\right) t}, \quad \text { with } m \in \mathbb{Z} \text { and }(p, q) \in\{-1,0,1\} .
$$

The restriction on the range of values for $p$ and $q$ comes from the fact that we are limited to the linear order in spins. This general structure will also be that of the time dependence of any of the source multipole moments, so that we shall be able to integrate the tail integrals using a simple formula in the Fourier domain.

Finally, we turn to the leading PN order of precession effects. A superficial look at Eqs. $3.22,3.20$ and $(3.12)$ would tell us that precession effects in the dynamical solution for the moving basis starts at order $\mathcal{O}\left(c^{-1}\right)$, which is the order of the first spin contribution in the angular momentum $\boldsymbol{J}$. However, we notice that only the combination $\sin \iota e^{\mathrm{i} \alpha}-\sin \iota_{0} e^{\mathrm{i} \alpha_{0}}$ and its complex conjugate intervene into the solution (3.22). At leading order, since $J_{+}=$ $\left(S_{n}+\mathrm{i} S_{\lambda}\right) / c+\mathcal{O}\left(c^{-3}\right)$, and using $\left|\boldsymbol{L}_{\mathrm{NS}}\right|=G m^{2} \nu /\left(c x^{1 / 2}\right)+\mathcal{O}\left(c^{-2}\right)$, we have

$$
\sin \iota e^{\mathrm{i} \alpha}=-\mathrm{i} \frac{x^{1 / 2}}{G m^{2} \nu} S_{\perp}^{1} e^{\mathrm{i}\left(\phi-\psi_{1}\right)}+1 \leftrightarrow 2+\mathcal{O}\left(\frac{1}{c^{3}}\right)
$$




$$
=-\mathrm{i} \frac{x^{1 / 2}}{G m^{2} \nu} S_{\perp}^{1} e^{\mathrm{i}\left[\phi_{0}-\psi_{0}^{1}+\Omega_{1}\left(t-t_{0}\right)\right]}+1 \leftrightarrow 2+\mathcal{O}\left(\frac{1}{c^{3}}\right),
$$

where $1 \leftrightarrow 2$ means the expression obtained by the exchange of the two particles. Now, by Taylor-expanding around the reference time $t_{0}$, we find that the combination $\sin \iota e^{\mathrm{i} \alpha}-$ $\sin \iota_{0} e^{\mathrm{i} \alpha_{0}}$ is made of terms proportional to $\Omega_{1} / c$ or $\Omega_{2} / c$ and therefore is of order $\mathcal{O}\left(c^{-3}\right)$, since the spin precession frequencies $\Omega_{A}$ are small and known to be already of 1PN order; $c f$. Eq. (3.14). Thus, we see that the precession effects due to the spins in our solution (3.22) are in fact of order $\mathcal{O}\left(c^{-3}\right)$ or $1.5 \mathrm{PN}$, as one could expect from their corresponding order in the acceleration.

\section{TAIL-INDUCED SPIN ORBIT EFFECTS IN THE FLUX}

The spin-orbit couplings in the relevant source moments $I_{L}$ and $J_{L}$ have been computed in Paper II up to next-to-next-to-leading order. To compute the $4 \mathrm{PN}$ spin-orbit tail contributions, we will need the mass and current quadrupole moments $I_{i j}$ and $J_{i j}($ with $\ell=2$ ) at relative order $1 \mathrm{PN}$ (for both the spin-orbit terms and the non-spin ones), and the mass and current octupoles $I_{i j k}$ and $J_{i j k}(\ell=3)$ at Newtonian order. The non-spin terms are well known at the corresponding $1 \mathrm{PN}$ order, see e.g. Ref. [44]. However, we point out that we need for this computation not only the quadrupole and octupole moments at $1 \mathrm{PN}$ order, but also the mass monopole $M$ at $1 \mathrm{PN}$ order, since this is that mass monopole which is responsible for the tails in Eqs. (2.3). The 1PN non-spin monopole for circular orbits reads

$$
M=m\left(1-\frac{\nu}{2} x\right)+\mathcal{O}\left(\frac{1}{c^{4}}\right) .
$$

Similarly we need also to include the spin-orbit terms into the mass monopole moment $M$. Remind that $M=m+E / c^{2}$ where $E$ is the conservative energy associated with the equations of motion. The spin-orbit effects in $E$ arise at $1.5 \mathrm{PN}$ order and have been given in Eqs. (3.9) of Ref. [2]. This means that the dominant spin-orbit effect in $M$ is not at order $1.5 \mathrm{PN}$ but rather at order $2.5 \mathrm{PN}$; for the present computation we need only the dominant 2.5PN spin-orbit term given by

$$
\underset{\mathrm{S}}{M}=\frac{G m \nu}{c^{5} r^{2}}\left\{-(n, S, v)-\frac{\delta m}{m}(n, \Sigma, v)\right\}+\mathcal{O}\left(\frac{1}{c^{7}}\right) .
$$

The spin-orbit contribution is indicated by a subscript $\mathrm{S}$ and we give the result already reduced to the center-of-mass frame. For the other moments we shall simply report the results taken from Paper II:

$$
\begin{aligned}
I_{\mathrm{S}} i j= & \frac{r \nu}{c^{3}}\left\{-\frac{8}{3}(\mathbf{S} \times \boldsymbol{v})^{<i} n^{j>}-\frac{8}{3} \frac{\delta m}{m}(\boldsymbol{\Sigma} \times \boldsymbol{v})^{<i} n^{j>}\right. \\
& \left.-\frac{4}{3}(\boldsymbol{n} \times \mathbf{S})^{<i} v^{j>}-\frac{4}{3} \frac{\delta m}{m}(\boldsymbol{n} \times \boldsymbol{\Sigma})^{<i} v^{j>}\right\} \\
+ & \frac{r \nu}{c^{5}}\left[\left\{(\mathbf{S} \times \boldsymbol{v})^{<i} n^{j>}\left(-\frac{26}{21}+\frac{26}{7} \nu\right) v^{2}+(\boldsymbol{\Sigma} \times \boldsymbol{v})^{<i} n^{j>} \frac{\delta m}{m}\left(-\frac{26}{21}+\frac{116}{21} \nu\right) v^{2}\right.\right.
\end{aligned}
$$




$$
\begin{aligned}
& +(\boldsymbol{n} \times \mathbf{S})^{<i} v^{j>}\left(-\frac{4}{21}+\frac{4}{7} \nu\right) v^{2}+(\boldsymbol{n} \times \boldsymbol{\Sigma})^{<i} v^{j>} \frac{\delta m}{m}\left(-\frac{4}{21}+\frac{12}{7} \nu\right) v^{2} \\
& +(\mathbf{S} \times \boldsymbol{v})^{<i} v^{j>}\left(\frac{4}{21}-\frac{4}{7} \nu\right)(n v)+(\boldsymbol{\Sigma} \times \boldsymbol{v})^{<i} v^{j>} \frac{\delta m}{m}\left(\frac{4}{21}-\frac{20}{21} \nu\right)(n v) \\
& \left.+(n, S, v) v^{<i} v^{j>}\left(-\frac{3}{7}+\frac{9}{7} \nu\right)+(n, \Sigma, v) v^{<i} v^{j>} \frac{\delta m}{m}\left(-\frac{3}{7}+\frac{40}{21} \nu\right)\right\} \\
& +\frac{G m}{r}\left\{(n, S, v) n^{<i} n^{j>}\left(-\frac{38}{21}-\frac{4}{7} \nu\right)+(n, \Sigma, v) n^{<i} n^{j>} \frac{\delta m}{m}\left(-\frac{16}{7}+\frac{26}{21} \nu\right)\right. \\
& +(\boldsymbol{n} \times \mathbf{S})^{<i} n^{j>}\left(\frac{17}{21}+\frac{61}{21} \nu\right)(n v)+(\boldsymbol{n} \times \boldsymbol{\Sigma})^{<i} n^{j>} \frac{\delta m}{m}\left(1+\frac{34}{21} \nu\right)(n v) \\
& +(n S)(\boldsymbol{n} \times \boldsymbol{v})^{<i} n^{j>}\left(-2+\frac{10}{3} \nu\right)+(n \Sigma)(\boldsymbol{n} \times \boldsymbol{v})^{<i} n^{j>} \frac{\delta m}{m}\left(-2+\frac{4}{3} \nu\right) \\
& +(\mathbf{S} \times \boldsymbol{v})^{<i} n^{j>}\left(-\frac{11}{7}-\frac{125}{21} \nu\right)+(\boldsymbol{\Sigma} \times \boldsymbol{v})^{<i} n^{j>} \frac{\delta m}{m}\left(-\frac{1}{3}-\frac{16}{3} \nu\right) \\
& \left.\left.+(\boldsymbol{n} \times \mathbf{S})^{<i} v^{j>}\left(-\frac{22}{3}-\frac{10}{3} \nu\right)+(\boldsymbol{n} \times \boldsymbol{\Sigma})^{<i} v^{j>} \frac{\delta m}{m}\left(-\frac{8}{3}-\frac{34}{21} \nu\right)\right\}\right]+\mathcal{O}\left(\frac{1}{c^{7}}\right) \\
& J_{\mathrm{S}} i j=\frac{r \nu}{c}\left\{-\frac{3}{2} \Sigma^{<i} n^{j>}\right\} \\
& +\frac{r \nu}{c^{3}}\left[\left\{-\frac{2}{7} \frac{\delta m}{m} v^{2} S^{<i} n^{j>}+\Sigma^{<i} n^{j>}\left(-\frac{29}{28}+\frac{143}{28} \nu\right) v^{2}\right.\right. \\
& +\frac{33}{28} \frac{\delta m}{m}(S v) n^{<i} v^{j>}+(\Sigma v) n^{<i} v^{j>}\left(\frac{33}{28}-\frac{155}{28} \nu\right) \\
& +\frac{3}{7} \frac{\delta m}{m}(n v) S^{<i} v^{j>}+\Sigma^{<i} v^{j>}\left(\frac{3}{7}-\frac{16}{7} \nu\right)(n v) \\
& \left.-\frac{11}{14} \frac{\delta m}{m}(n S) v^{<i} v^{j>}+(n \Sigma) v^{<i} v^{j>}\left(-\frac{11}{14}+\frac{47}{14} \nu\right)\right\} \\
& +\frac{G m}{r}\left\{-\frac{29}{14} \frac{\delta m}{m}(n S) n^{<i} n^{j>}+(n \Sigma) n^{<i} n^{j>}\left(-\frac{4}{7}+\frac{31}{14} \nu\right)\right. \\
& \left.\left.+\frac{10}{7} \frac{\delta m}{m} S^{<i} n^{j>}+\Sigma^{<i} n^{j>}\left(\frac{61}{28}-\frac{71}{28} \nu\right)\right\}\right]+\mathcal{O}\left(\frac{1}{c^{5}}\right) \\
& {\underset{\mathrm{S}}{i j k}}_{i j k}=\frac{r^{2} \nu}{c^{3}}\left\{\frac{9}{2} \frac{\delta m}{m}(\mathbf{S} \times \boldsymbol{v})^{<i} n^{j} n^{k>}+(\boldsymbol{\Sigma} \times \boldsymbol{v})^{<i} n^{j} n^{k>}\left(\frac{9}{2}-\frac{33}{2} \nu\right)\right. \\
& \left.+3 \frac{\delta m}{m}(\boldsymbol{n} \times \mathbf{S})^{<i} n^{j} v^{k>}+(\boldsymbol{n} \times \boldsymbol{\Sigma})^{<i} n^{j} v^{k>}(3-9 \nu)\right\}+\mathcal{O}\left(\frac{1}{c^{5}}\right), \\
& J_{\mathrm{S}} i j k=\frac{r^{2} \nu}{c}\left\{2 S^{<i} n^{j} n^{k>}+2 \frac{\delta m}{m} \Sigma^{<i} n^{j} n^{k>}\right\}+\mathcal{O}\left(\frac{1}{c^{3}}\right) .
\end{aligned}
$$

We recall that these spin parts of multipole moments are expressed in terms of the conservedmagnitude spins and of the useful variables (3.1). We recall also our notation, e.g. $(v S) \equiv$ 
$\boldsymbol{v} \cdot \mathbf{S}$ for the ordinary Euclidean scalar product, $(\boldsymbol{x} \times \boldsymbol{\Sigma})^{i} \equiv \varepsilon^{i j k} x^{j} \Sigma^{k}$ for the ordinary cross product, and $(S, x, v) \equiv \mathbf{S} \cdot(\boldsymbol{x} \times \boldsymbol{v})=\varepsilon^{i j k} S^{i} x^{j} v^{k}$ for the mixed product.

We now turn to the calculation of the tail integrals (2.3), where, as we have already shown, we can replace the canonical moments $M_{L}, S_{L}$ by the source moments $I_{L}, J_{L}$. Following Paper I, we found more convenient to do this computation in the Fourier domain. We denote by $K_{L}$ a generic source moment $I_{L}$ or $J_{L}$, and we define its Fourier transform as

$$
K_{L}(t)=\int_{-\infty}^{+\infty} \frac{\mathrm{d} \Omega}{2 \pi} \tilde{K}_{L}(\Omega) e^{-\mathrm{i} \Omega t}, \quad \tilde{K}_{L}(\Omega)=\int_{-\infty}^{+\infty} \mathrm{d} t K_{L}(t) e^{\mathrm{i} \Omega t} .
$$

It was shown in Ref. [47] (see also Sec. II B in Paper I) that, under the assumption that the binary formed in the remote past from some quasi-hyperbolic orbits by gravitational radiation, a generic integral of the form

$$
\mathcal{U}_{L}\left(T_{R}\right) \equiv \int_{0}^{+\infty} \mathrm{d} \tau K_{L}^{(\ell+2)}\left(T_{R}-\tau\right) \ln \left(\frac{\tau}{2 \hat{\tau}_{0}}\right)
$$

where $\hat{\tau}_{0}$ means either $\tau_{0} e^{-\kappa_{\ell}}$ or $\tau_{0} e^{-\pi_{\ell}}$, takes the following expression in the Fourier domain:

$$
\mathcal{U}_{L}\left(T_{R}\right)=\mathrm{i} \int_{-\infty}^{+\infty} \frac{\mathrm{d} \Omega}{2 \pi}(-\mathrm{i} \Omega)^{\ell+1} \tilde{K}_{L}(\Omega) e^{-\mathrm{i} \Omega T_{R}}\left[\frac{\pi}{2} s(\Omega)+\mathrm{i}\left(\ln \left(2|\Omega| \hat{\tau}_{0}\right)+\gamma_{\mathrm{E}}\right)\right],
$$

where $s(\Omega)$ is the sign of $\Omega$ and $\gamma_{\mathrm{E}}$ is the Euler constant. Now, given the general structure of the frequency modes $(3.25)$, we see that the Fourier coefficients $\tilde{K}_{L}(\Omega)$ consist of a finite sum over frequencies,

$$
\tilde{K}_{L}(\Omega)=2 \pi \sum_{m, p, q} A_{L}^{m, p, q} \delta\left(\Omega-\omega_{m, p, q}\right)
$$

in which $\omega_{m, p, q}=m \omega+p \Omega_{1}+q \Omega_{2}$, and where the sum is finite, limited to $-1 \leqslant p, q \leqslant 1$ and with $m$ taking a finite number of integer values (depending on the order of approximation). The amplitudes $A_{L}^{m, p, q}$ can be readily read off the explicit expressions of the source moments. Then Eq. (4.6) transforms into

$$
\mathcal{U}_{L}\left(T_{R}\right)=\mathrm{i} \sum_{m, p, q} A_{L}^{m, p, q}\left(-\mathrm{i} \omega_{m, p, q}\right)^{\ell+1} e^{-\mathrm{i} \omega_{m, p, q} T_{R}}\left[\frac{\pi}{2} s\left(\omega_{m, p, q}\right)+\mathrm{i}\left(\ln \left(2\left|\omega_{m, p, q}\right| \hat{\tau}_{0}\right)+\gamma_{\mathrm{E}}\right)\right] .
$$

When applying this formula, in agreement with the dimensional argument presented in Sec. II B, we find that the constant $\hat{\tau}_{0}$ cancels out in the flux (and so does $\gamma_{E}$ ). It also turns out that the various precessional corrections cancel out. That is to say, ignoring the precessional contributions given by the second terms in Eqs. (3.22) would yield the same final result for the flux. This is due to the fact that we are computing a scalar, and can be explained by a structural argument presented in Appendix A.

Finally, we give our main result for the emitted energy flux of quasi-circular orbits. The spin-orbit part of the flux up to $4 \mathrm{PN}$ order, thus including the new next-to-leading $4 \mathrm{PN}$ tail-induced term, reads

$$
\begin{aligned}
\underset{\mathrm{S}}{\mathcal{F}}=\frac{32 c^{5}}{5 G} x^{5} \nu^{2}\left(\frac{x^{3 / 2}}{G m^{2}}\right)\left\{-4 S_{\ell}-\frac{5}{4} \frac{\delta m}{m} \Sigma_{\ell}\right. \\
+x\left[\left(-\frac{9}{2}+\frac{272}{9} \nu\right) S_{\ell}+\left(-\frac{13}{16}+\frac{43}{4} \nu\right) \frac{\delta m}{m} \Sigma_{\ell}\right]
\end{aligned}
$$




$$
\begin{aligned}
& +x^{3 / 2}\left[-16 \pi S_{\ell}-\frac{31 \pi}{6} \frac{\delta m}{m} \Sigma_{\ell}\right] \\
& +x^{2}\left[\left(\frac{476645}{6804}+\frac{6172}{189} \nu-\frac{2810}{27} \nu^{2}\right) S_{\ell}+\left(\frac{9535}{336}+\frac{1849}{126} \nu-\frac{1501}{36} \nu^{2}\right) \frac{\delta m}{m} \Sigma_{\ell}\right] \\
& +x^{5 / 2}\left[\left(-\frac{3485 \pi}{96}+\frac{13879 \pi}{72} \nu\right) S_{\ell}+\left(-\frac{7163 \pi}{672}+\frac{130583 \pi}{2016} \nu\right) \frac{\delta m}{m} \Sigma_{\ell}\right] \\
& \left.+\mathcal{O}\left(\frac{1}{c^{6}}\right)\right\} .
\end{aligned}
$$

As usual, the spin-orbit contributions due to the absorption by the black-hole horizons have to be added to the post-Newtonian result computed here [48 51. The result (4.9) for the spin-orbit contribution to the energy flux is to be added to the non-spin contributions given up to $3.5 \mathrm{PN}$ by Eq. (230) in Ref. [40]. The spin-spin effects in the flux are known to leading order from Refs. [20, 21, 52,

We have also derived the 4PN tail-induced terms in the energy flux through an alternative, but equivalent computation that uses Eq. (2.9) in Ref. [53] extended through 4PN order (i.e. we have added also the term that involves the current octupole moment). For this derivation we have worked in the time domain, computed derivatives of the relevant multipole moments, reduced to quasi-circular orbits and then calculated the tail integrals in the complex plane, e.g., as described in Sec. IVB and Appendix C of Ref. [54]. Moreover, quite satisfactorily, the result (4.9) is in complete agreement in the test-mass limit where $\nu \rightarrow 0$ with the result of black-hole perturbation theory on a Kerr background [55].

To obtain the evolution of the orbital phase for quasi-circular orbits we apply like in Papers I \& II the usual energy balance equation. The conservative energy $E$ in the balance equation does not contain any spin-orbit term at 4PN order - this can be seen dimensionally like for the absence of instantaneous terms in the flux. Therefore it is the same as used in Paper II (and was computed at the right order in the previous works [1, 2]). We obtain the secular evolution of the orbital frequency $\omega$ and carrier phase $\phi \equiv \int \omega \mathrm{d} t$ as

$$
\begin{aligned}
\left(\frac{\dot{\omega}}{\omega^{2}}\right)_{\mathrm{S}}=\frac{96}{5} & \nu x^{5 / 2}\left(\frac{x^{3 / 2}}{G m^{2}}\right)\left\{-\frac{47}{3} S_{\ell}-\frac{25}{4} \frac{\delta m}{m} \Sigma_{\ell}\right. \\
& +x\left[\left(-\frac{5861}{144}+\frac{1001}{12} \nu\right) S_{\ell}+\left(-\frac{809}{84}+\frac{281}{8} \nu\right) \frac{\delta m}{m} \Sigma_{\ell}\right] \\
& +x^{3 / 2}\left[-\frac{188 \pi}{3} S_{\ell}-\frac{151 \pi}{6} \frac{\delta m}{m} \Sigma_{\ell}\right] \\
& +x^{2}\left[\left(-\frac{4323559}{18144}+\frac{436705}{672} \nu-\frac{5575}{27} \nu^{2}\right) S_{\ell}\right. \\
& +x^{5 / 2}\left[\left(-\frac{15271 \pi}{72}+\frac{3317 \pi}{6} \nu\right) S_{\ell}+\left(-\frac{1665 \pi}{28}+\frac{50483 \pi}{224} \nu\right) \frac{\delta m}{m} \Sigma_{\ell}\right] \\
& \left.+\mathcal{O}\left(\frac{1}{c^{6}}\right)\right\} . \\
\phi=- & \frac{x^{-5 / 2}}{32 \nu}\left(\frac{x^{3 / 2}}{G m^{2}}\right)\left\{\frac{235}{6} S_{\ell}+\frac{125}{8} \frac{\delta m}{m} \Sigma_{\ell}\right.
\end{aligned}
$$




$$
\begin{aligned}
& +x \ln x\left[\left(-\frac{554345}{2016}-\frac{55}{8} \nu\right) S_{\ell}+\left(-\frac{41745}{448}+\frac{15}{8} \nu\right) \frac{\delta m}{m} \Sigma_{\ell}\right] \\
& +x^{3 / 2}\left[\frac{940 \pi}{3} S_{\ell}+\frac{745 \pi}{6} \frac{\delta m}{m} \Sigma_{\ell}\right] \\
& +x^{2}\left[\left(-\frac{8980424995}{6096384}+\frac{6586595}{6048} \nu-\frac{305}{288} \nu^{2}\right) S_{\ell}\right. \\
& \left.\quad+\left(-\frac{170978035}{387072}+\frac{2876425}{5376} \nu+\frac{4735}{1152} \nu^{2}\right) \frac{\delta m}{m} \Sigma_{\ell}\right] \\
& +x^{5 / 2}\left[\left(\frac{2388425 \pi}{3024}-\frac{9925 \pi}{36} \nu\right) S_{\ell}+\left(\frac{3237995 \pi}{12096}-\frac{258245 \pi}{2016} \nu\right) \frac{\delta m}{m} \Sigma_{\ell}\right] \\
& \left.+\mathcal{O}\left(\frac{1}{c^{6}}\right)\right\} .
\end{aligned}
$$

The expressions $(4.9)$ and 4.10 constitute the main theoretical inputs needed for the construction of gravitational wave templates. The non-spin terms in the carrier phase can be found in Eq. (235) of Ref. [40], and those in $\dot{\omega} / \omega^{2}$ in e.g. Eq. (32) of Ref. [56]. However, recall that in the case of precessional binaries we must add to the carrier phase $\phi$ the precessional correction arising from the precession of the orbital plane, namely $\Phi=\phi-\alpha$ in the notation of Eq. (3.18). For this precessional correction one can use directly the results of Sec. IIIB.

As an illustration of the significance of the new terms, we show in the Table I the contribution of each post-Newtonian order to the number of accumulated gravitational-wave cycles, computed using the so-called Taylor T2 approximant. For neutron star or stellar mass black hole binaries targeted by ground-based detectors similar to LIGO and Virgo, the number of cycles is between a minimal frequency corresponding to a seismic noise cut-off at $10 \mathrm{~Hz}$ and a maximal frequency taken to be the Schwarzschild ISCO frequency $\omega_{\max }=\omega_{\text {ISCO }}=c^{3} /\left(6^{3 / 2} G m\right)$. Recall that the parameter $\chi$ is small for a neutron star but can be close to one for astrophysical black holes [15].

As we see, the $4 \mathrm{PN}$ spin-orbit terms computed in the present paper can be significant and

\begin{tabular}{|r|c|c|c|}
\hline LIGO/Virgo & $1.4 M_{\odot}+1.4 M_{\odot}$ & $10 M_{\odot}+1.4 M_{\odot}$ & $10 M_{\odot}+10 M_{\odot}$ \\
\hline \hline Newtonian & 15952.6 & 3558.9 & 598.8 \\
$1 \mathrm{PN}$ & 439.5 & 212.4 & 59.1 \\
$1.5 \mathrm{PN}$ & $-210.3+65.6 \kappa_{1} \chi_{1}+65.6 \kappa_{2} \chi_{2}$ & $-180.9+114.0 \kappa_{1} \chi_{1}+11.7 \kappa_{2} \chi_{2}$ & $-51.2+16.0 \kappa_{1} \chi_{1}+16.0 \kappa_{2} \chi_{2}$ \\
$2 \mathrm{PN}$ & 9.9 & 9.8 & 4.0 \\
$2.5 \mathrm{PN}$ & $-11.7+9.3 \kappa_{1} \chi_{1}+9.3 \kappa_{2} \chi_{2}$ & $-20.0+33.8 \kappa_{1} \chi_{1}+2.9 \kappa_{2} \chi_{2}$ & $-7.1+5.7 \kappa_{1} \chi_{1}+5.7 \kappa_{2} \chi_{2}$ \\
$3 \mathrm{PN}$ & $2.6-3.2 \kappa_{1} \chi_{1}-3.2 \kappa_{2} \chi_{2}$ & $2.3-13.2 \kappa_{1} \chi_{1}-1.3 \kappa_{2} \chi_{2}$ & $2.2-2.6 \kappa_{1} \chi_{1}-2.6 \kappa_{2} \chi_{2}$ \\
$3.5 \mathrm{PN}$ & $-0.9+1.9 \kappa_{1} \chi_{1}+1.9 \kappa_{2} \chi_{2}$ & $-1.8+11.1 \kappa_{1} \chi_{1}+0.8 \kappa_{2} \chi_{2}$ & $-0.8+1.7 \kappa_{1} \chi_{1}+1.7 \kappa_{2} \chi_{2}$ \\
$4 \mathrm{PN}$ & $(\mathrm{NS})-1.5 \kappa_{1} \chi_{1}-1.5 \kappa_{2} \chi_{2}$ & $(\mathrm{NS})-8.0 \kappa_{1} \chi_{1}-0.7 \kappa_{2} \chi_{2}$ & $(\mathrm{NS})-1.5 \kappa_{1} \chi_{1}-1.5 \kappa_{2} \chi_{2}$ \\
\hline
\end{tabular}

TABLE I. Spin-orbit contributions to the number of gravitational-wave cycles $\mathcal{N}_{\mathrm{GW}}=\left(\phi_{\max }-\right.$ $\left.\phi_{\min }\right) / \pi$. For binaries detectable by ground-based detectors LIGO/Virgo, we show the number of cycles accumulated from $\omega_{\min }=\pi \times 10 \mathrm{~Hz}$ to $\omega_{\max }=\omega_{\text {ISCO }}=c^{3} /\left(6^{3 / 2} G m\right)$. For each compact object we define the magnitude $\chi_{A}$ and the orientation $\kappa_{A}$ of the spin by $\mathbf{S}_{A} \equiv G m_{A}^{2} \chi_{A} \hat{\mathbf{S}}_{A}$ and $\kappa_{A} \equiv \hat{\mathbf{S}}_{A} \cdot \boldsymbol{\ell}$. For comparison, we give all the non-spin contributions up to 3.5PN order, but the non-spin $4 \mathrm{PN}$ terms (NS) are yet unknown. We neglect all the spin-spin terms. 
are worth to be included in the gravitational wave templates. In particular, these terms are comparable, although a bit smaller, to the previous $3.5 \mathrm{PN}$ spin-orbit terms. Interestingly, notice that in fact the $4 \mathrm{PN}$ terms tend to significantly cancel out numerically the contributions of the $3.5 \mathrm{PN}$ terms. At the $3.5 \mathrm{PN}$ order the effect of spin-orbit terms can be larger than the effect of the non-spinning terms, especially in the case of asymmetric binaries. At the $4 \mathrm{PN}$ order we do not know if this happens since the $4 \mathrm{PN}$ non-spin terms have not yet been computed.

We emphasize that it will be important in the future to improve the knowledge of the phasing by computing spin-spin and even spin-spin-spin terms through at least 4PN and 3.5PN order, respectively, and also spin effects induced by the black-hole's horizon-absorbed energy flux [48 51]. Those terms may give a contribution to the phasing of the same order as the one computed in this paper, especially when the black holes carry large spins and the orbit approaches the ISCO.

As a last comment, one should obviously keep in mind that the numerical results in Table II only give an illustration of the order of magnitude of the various contributions. Indeed the precise analysis should take into account the details of the noise spectral density of the detectors, and one should focus on studying the incidence of the various contributions on the parameter estimation rather than simply counting the number of cycles. In addition, note that the numerical values reported in Table I depend on the type of approximant that one uses, here the T2 approximant. We find that using the Taylor T1 and Taylor T4 approximants leads to similar conclusions for our new $4 \mathrm{PN}$ tail contribution, i.e. a variation of the order of one or a few cycles for maximally spinning black holes.

\section{ACKNOWLEDGEMENTS}

It is a pleasure to thank Guillaume Faye for discussions. A. Bohé is grateful for the support of the Spanish MIMECO grant FPA2010-16495, the European Union FEDER funds, and the Conselleria d'Economia i Competitivitat del Govern de les Illes Balears. A. Buonanno acknowledges partial support from NSF Grant No. PHY-1208881 and NASA Grant

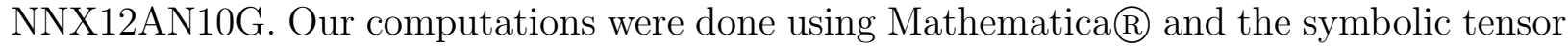
calculus package xAct [57].

\section{Appendix A: Cancellation of precessional contributions in the flux}

In this Appendix, we explain why the precessional contributions in the evolution of the moving triad, given by the second terms in the right-hand sides of Eq. (3.22), identically cancel in the final flux at linear order in spin. Let us consider the structure of the contributions of the tail terms in the flux. From (2.2), (2.3) and (2.6), we get that these contributions take the form:

$$
K_{L}^{(\ell+1)}\left(T_{R}\right) \frac{2 G M}{c^{3}} \int_{0}^{+\infty} \mathrm{d} \tau K_{L}^{(\ell+3)}\left(T_{R}-\tau\right) \ln \left(\frac{\tau}{2 \hat{\tau}_{0}}\right)
$$

where $K_{L}$ is indifferently a source moment $I_{L}$ or $J_{L}$. In the following, we will refer to $\left(\boldsymbol{n}_{0}, \boldsymbol{\lambda}_{0}, \boldsymbol{\ell}_{0}\right)$ as the moving triad evaluated at time $T_{R}$. When expressing the time derivatives

$K_{L}^{(\ell+1)}$ and $K_{L}^{(\ell+3)}$ projected on the moving basis, we obtain an explicit spin-dependent part and a "non-spin" part which depends on the spins only implicitly through the vectors $(\boldsymbol{n}, \boldsymbol{\lambda}, \boldsymbol{\ell})$. The spin part already displays a spin vector and, since the precessional terms 
in (3.22) are linear in spin, we can ignore them at the spin-orbit level and use the first terms in (3.22), which correspond to the nonprecessional dynamics. Thus, we only have to consider the non-spin part of Eq. (A1), and look at the implicit spin dependence through the evolution of $(\boldsymbol{n}, \boldsymbol{\lambda}, \boldsymbol{\ell})$.

Now, the non-spin contributions to the source moments, as given for instance in Ref. [44, and their derivatives, will display only the vectors $\boldsymbol{n}$ and $\boldsymbol{\lambda}$, but not the vector $\boldsymbol{\ell}$; there will also be a Levi-Civita symbol $\varepsilon_{i j k}$ for current-type moments, which we keep explicitly for the argument. At the order considered here, we need only to consider $I_{i j}$ at 1PN order, and $J_{i j}$ and $I_{i j k}$ at Newtonian order. We can expand their expressions using Eq. (3.22a and apply the Fourier-domain formula (4.8) for the term under the integral. The point is that the vectorial structure is kept the same: the part that is proportional to $\boldsymbol{\ell}_{0}$ contains a spin and comes from the second term of $\boldsymbol{m}$ in (3.22a). When considering the contraction of the two terms of Eq. A1), inside and outside the integral, to produce a scalar, we are left with a combination of contractions of the basis vectors $\left(\boldsymbol{n}_{0}, \boldsymbol{\lambda}_{0}, \boldsymbol{\ell}_{0}\right)$ (for current-type moments, a product of Levi-Civita symbols appears, which reduces to a sum of products of Kronecker deltas). The term outside the integral contains only the vectors $\boldsymbol{n}_{0}, \boldsymbol{\lambda}_{0}$, and the precessional "non-spin" term inside the integral is proportional to the vector $\boldsymbol{\ell}_{0}$ at linear order in spin. In the contraction, this vector is forced to enter a scalar product with $\boldsymbol{n}_{0}$ or $\boldsymbol{\lambda}_{0}$, and the contribution cancels out. This argument, as the other results of this paper, is only valid at linear order in spin.

However, notice that the precessional contributions will obviously not cancel out in the individual radiative moments, and therefore will affect the waveform, as already found in Paper I. Hence, the general calculations that we have explained in Sec. III, including precession, will be useful for future investigations of the waveform.

[1] S. Marsat, A. Bohé, G. Faye, and L. Blanchet, Class. Quant. Grav. 30, 055007 (2013), 1210.4143 .

[2] A. Bohé, S. Marsat, G. Faye, and L. Blanchet, Class. Quant. Grav. 30, 075017 (2013), 1212.5520 .

[3] A. Bohé, S. Marsat, and L. Blanchet, Class. Quant. Grav. (2013), 1303.7412.

[4] J. Hartung and J. Steinhoff, Annalen der Physik 523, 783 (2011), arXiv:1104.3079 [gr-qc],

[5] J. Hartung, J. Steinhoff, and G. Schäfer, Annalen der Physik (2013), arXiv:1302.6723 [gr-qc],

[6] L. Blanchet, A. Buonanno, and G. Faye, Phys.Rev. D84, 064041 (2011), arXiv:1104.5659 [gr-qc],

[7] M. A. Abramowicz and W. Kluźniak, Astron. Astrophys. 374, L19 (2001).

[8] T. E. Strohmayer, Astrophys. J. 552, L49 (2001).

[9] J. E. McClintock, R. Shafee, R. Narayan, R. A. Remillard, S. W. Davis, and L.-X. Li, Astrophys. J. 652, 518 (2006), arXiv:astro-ph/0606076.

[10] L. Gou, J. E. McClintock, M. J. Reid, J. A. Orosz, J. F. Steiner, R. Narayan, J. Xiang, R. A. Remillard, K. A. Arnaud, and S. W. Davis, Astrophys. J. 742, 85 (2011), arXiv:1106.3690 [astro-ph.HE],

[11] M. A. Nowak, J. Wilms, K. Pottschmidt, N. Schulz, J. Miller, and D. Maitra, in American Institute of Physics Conference Series, American Institute of Physics Conference Series, Vol. 1427, edited by R. Petre, K. Mitsuda, and L. Angelini (2012) pp. 48-51. 
[12] A. C. Fabian and G. Miniutti, in The Kerr Spacetime: Rotating Black Holes in General Relativity, edited by D. L. Wiltshire, M. Visser, and S. M. Scott (Cambridge U. Press, 2009) Chap. 9, arXiv:astro-ph/0507409.

[13] L. W. Brenneman and C. S. Reynolds, Astrophys. J. 652, 1028 (2006), arXiv:astro$\mathrm{ph} / 0608502$.

[14] L. W. Brenneman, C. S. Reynolds, M. A. Nowak, R. C. Reis, M. Trippe, A. C. Fabian, K. Iwasawa, J. C. Lee, J. M. Miller, R. F. Mushotzky, K. Nandra, and M. Volonteri, Astrophys. J. 736, 103 (2011), arXiv:1104.1172 [astro-ph.HE].

[15] C. S. Reynolds, (2013), arXiv:1302.3260 [astro-ph.HE],

[16] C. Cutler and E. Flanagan, Phys. Rev. D 49, 2658 (1994).

[17] T. Apostolatos, C. Cutler, G. Sussman, and K. Thorne, Phys. Rev. D 49, 6274 (1994).

[18] B. Barker and R. O'Connell, Phys. Rev. D 12, 329 (1975).

[19] B. Barker and R. O'Connell, Gen. Relativ. Gravit. 11, 149 (1979).

[20] L. Kidder, C. Will, and A. Wiseman, Phys. Rev. D 47, R4183 (1993).

[21] L. Kidder, Phys. Rev. D 52, 821 (1995), gr-qc/9506022.

[22] H. Tagoshi, A. Ohashi, and B. Owen, Phys. Rev. D 63, 044006 (2001).

[23] G. Faye, L. Blanchet, and A. Buonanno, Phys. Rev. D 74, 104033 (2006), gr-qc/0605139.

[24] T. Damour, P. Jaranowski, and G. Schäfer, Phys. Rev. D 77, 064032 (2008).

[25] M. Levi, Phys.Rev. D82, 104004 (2010), arXiv:1006.4139 [gr-qc].

[26] R. A. Porto, Classical and Quantum Gravity 27, 205001 (2010), arXiv:1005.5730 [gr-qc].

[27] L. Blanchet, A. Buonanno, and G. Faye, Phys. Rev. D 74, 104034 (2006), erratum Phys. Rev. D, 75:049903, 2007, gr-qc/0605140.

[28] W. D. Goldberger and I. Z. Rothstein, Phys. Rev. D 73, 104029 (2006), gr-qc/0409156.

[29] R. Porto, Phys. Rev. D 73, 104031 (2006), gr-qc/0511061.

[30] A. Buonanno, G. Faye, and T. Hinderer, Phys. Rev. D 87, 044009 (2013), 10.1103/PhysRevD.87.044009, arXiv:1209.6349 [gr-qc],

[31] S. Hergt, J. Steinhoff, and G. Schäfer, Class. Quant. Grav. 27, 135007 (2010), arXiv:1002.2093 [gr-qc].

[32] J. Steinhoff, S. Hergt, and G. Schäfer, Phys. Rev. D 78, 101503 (2008).

[33] R. A. Porto and I. Z. Rothstein, Phys.Rev. D78, 044012 (2008), arXiv:0802.0720 [gr-qc].

[34] R. A. Porto and I. Z. Rothstein, Phys.Rev. 78, 044013 (2008), arXiv:0804.0260 [gr-qc].

[35] M. Levi, Phys. Rev. D 82, 064029 (2010), arXiv:0802.1508 [gr-qc].

[36] J. Hartung and J. Steinhoff, Annalen der Physik 523, 919 (2011), arXiv:1107.4294 [gr-qc]

[37] M. Levi, Phys. Rev. D 85, 064043 (2012).

[38] L. Blanchet and T. Damour, Phil. Trans. Roy. Soc. Lond. A 320, 379 (1986).

[39] L. Blanchet, Class. Quant. Grav. 15, 1971 (1998), gr-qc/9801101.

[40] L. Blanchet, Living Rev. Rel. 9, 4 (2006), gr-qc/0202016.

[41] K. Thorne, Rev. Mod. Phys. 52, 299 (1980).

[42] L. Blanchet and T. Damour, Phys. Rev. D 46, 4304 (1992).

[43] L. Blanchet, Phys. Rev. D 51, 2559 (1995), gr-qc/9501030.

[44] L. Blanchet, G. Faye, B. R. Iyer, and S. Sinha, Class. Quant. Grav. 25, 165003 (2008), 0802.1249.

[45] G. Faye, S. Marsat, L. Blanchet, and B. R. Iyer, Class. Quant. Grav. 29, 175004 (2012), 1204.1043 .

[46] C. Will, Phys. Rev. D 71, 084027 (2005), gr-qc/0502039.

[47] L. Blanchet and G. Schäfer, Class. Quant. Grav. 10, 2699 (1993). 
[48] E. Poisson and M. Sasaki, Phys. Rev. D 51, 5753 (1995).

[49] K. Alvi, Phys. Rev. D 64, 104020 (2001), arXiv:0107080 [gr-qc].

[50] H. Tagoshi, S. Mano, and E. Takasugi, Prog.Theor.Phys. 98, 829 (1997), arXiv:gr-qc/9711072 $[\mathrm{gr}-\mathrm{qc}]$.

[51] K. Chatziioannou, E. Poisson, and N. Yunes, (2012), arXiv:1211.1686 [gr-qc]

[52] E. Poisson, Phys.Rev. D57, 5287 (1998), arXiv:gr-qc/9709032 [gr-qc],

[53] K. Arun, L. Blanchet, B. R. Iyer, and M. S. Qusailah, Phys. Rev. D 77, 064035 (2008).

[54] E. Racine, A. Buonanno, and L. E. Kidder, Phys.Rev. D80, 044010 (2009), arXiv:0812.4413 [gr-qc].

[55] H. Tagoshi, M. Shibata, T. Tanaka, and M. Sasaki, Phys. Rev. D 54, 1439 (1996).

[56] A. Buonanno, G. B. Cook, and F. Pretorius, Phys. Rev. D 75, 124018 (2007), gr-qc/0610122.

[57] J. M. Martín-García, A. García-Parrado, A. Stecchina, B. Wardell, C. Pitrou, D. Brizuela, D. Yllanes, G. Faye, L. Stein, R. Portugal, and T. Bäckdahl, "xAct: Efficient tensor computer algebra for Mathematica," (GPL 2002-2012), http://www.xact.es/. 